Aksaray University
Journal of Science and Engineering
e-ISSN: 2587-1277
http://dergipark.gov.tr/asujse
http://asujse.aksaray.edu.tr

Research Article

\title{
Assessment and Development of Path Loss Propagation Model for Ikire Metropolis, Nigeria
}

\author{
Jide J. Popoola* \\ Department of Electrical and Electronics Engineering, School of Engineeering and Engineering Technology, \\ Federal University of Technology, Akure, Nigeria
}

-Received Date: Jun 8, 2020 -Revised Date: Jun 15, 2021 -Accepted Date: Mar 25, 2021 -Published Online: Apr 21, 2021

\begin{abstract}
The aim of this paper is to assess and develop path loss propagation model for terrestial radio broadcasting station in Ikire, Nigeria. In carrying out the study, the reception quality of Osun State Corporation (OSBC) broadcast signal strength within Ikire metropolis in Irewole Local Government Area of Osun State, Nigeria was assessed using BC1173 Field Strength meter. The data obtained from the signal strength measurements around the metropolis were subsequently analysed and used for the development of an ideal propagation path loss model for the metropolis. The developed path loss model for the metropolis was evaluated and found to outperform the COST-231 Hata model in literature. The comparative performance evaluation result of the developed model and the COST-231 Hata model buttresses the need for developing different path loss models for different radio signals in different locations under different environmental factors. In addition, the finding of the study establishes a standard propagation path loss model that can be used for planning and designing efficient wireless communication link for terrestrial radio broadcasting station in the metropolis and any other environments with similar environmental factors.
\end{abstract}

\section{Keywords}

Radiowave, Path loss, Radiowave propagation, Radio propagation model

${ }^{*}$ Corresponding Author: Jide J. Popoola, jidejulius2001@gmail.com (DD0000-0001-9353-4447 


\section{INTRODUCTION}

Radiowave is a form of electromagnetic wave that travels through the space from the transmitter or sending end to the receiver or receiving end via the channel. The behaviour of radiowaves when transmitting from transmitter to receiver is known as radio propagation. Generally, radiowaves or broadcast signals during propagation are prone to losses due to many factors such as absorption, reflection, diffraction, refraction, polarization and scattering [1-3]. Similarly, daily variation in the quantity of water vapour present in the troposphere as well as the qunatity of ionization in the upper atmosphere as a result of sun does contribute to signal degradation.

Generally, during radiowave propagation, signal degradation or reduction in signal strength usually occurs due to path loss, which according to [4] is a key element in testing and designing of the link budget of a wireless system. Path loss, by simple definition is a reduction experienced by an electromagnetic wave during transmission from a transmitter to a receiver in a communication system. It is a phenomenon that occurs when the broadcast signal received becomes weak with respect to increase in distance between transmitter and receiver. Apart from absorption, reflection, diffraction, refraction, polarization, scattering and propagation medium (dry or moist air), path loss also occurs as a result of terrain contours, environment (urban or rural, vegetation and foliage), the distance between the transmitter and the receiver, and the height and location of antennas [5-7]. Thus, path loss encountered along any radio link serves as the dominant factor for characterization of propagation for the link.

Thus, according to [6], radio propagation model is an emprical mathematical formulation for the classification of radiowave propagation as function of distance, frequency and other conditions that regulates the behaviour of radio signal in a given transmission channel. Since individual wireless communication system encounters different terrain, obstructions and atmospheric conditions based on where they are propagated, it is not sufficient to formulate a single mathematical equation to model all factors in all environs and terrains $[8,9]$. Hence, in order to improve the strength of the transmitted signal in wireless channel, there is need to evaluate the path loss introduced by a terrain to adequately compensate for the signal power loss during signal transmission. This makes received signal path loss prediction models essential in wireless communication. As reported in [10], accurate path loss predictions models are used to find broadcast signal gaps and areas with poor serviceability. This accounts for why path loss propagation models are essential in signal prediction, planning, coverage and optimization. Furtheremore, path loss propagation models are also being used for interference 
analysis in order to ensure coexistence between services especially in cellular or mobile communication systems, fixed wireless access system and radio and television broadcasting [11].

Therefore, in this study, path loss prediction approach was employed in determining the coverage efficiency of OSBC in Ikire metropolis of Osun State so that the primary aim of establishing OSBC in disseminating socio-economic quality information with quality reception potential to all the dwellers of the state can be achieved. However due to the poor reception of OSBC radio signal in the metropolis, this study was embarked upon with aims of: (i) assessing the behavioural pattern of the station's signal in the metropolis; and (ii) developing a path loss model that can improve the station signal reception and any other similar locations with similar terrain in other parts of the world. In achieving these primary aims, the following objectives were set. One, to conduct signal field strength measurement of OSBC radio signal in the metropolis. Two, to develop a unique radiowave propagation model for the metropolis based on data obtained from objective one. Three, to evaluate the performance of the developed radiowave propagation model in objective two.

In ensuring chronological and coherent presentation of the study, the remaining parts of this paper are organised as follows. In Section 2, brief review of some related studies on radiowave propagation and path loss models were presented. In Section 3, the methodology involved in carrying out this study was presented. The results obtained were presented and discussed in Section 4. The conclusion was presented in Section 5, which is the last section of the paper.

\section{REVIEW OF RADIOWAVE PROPAGATION AND PATH LOSS MODELS}

Radiowave propagation as defined in [9] is the behaviour of radiowaves when they are propagated or transmitted from one point on the earth to another point or into various parts of the atmosphere. It is also defined as the way and manner radiowave travels between the transmitter and receiver, which is generally done either directly from one point to another or following the curvature of the earth or becoming trapped in the atmosphere and travelling longer distance or refracting off the ionosphere back to earth. Irrespective of the propagation mode, radiowave propagation is always affected by different phenomenon [9, 12-17] leading to path loss or signal degradation.

In overcoming the path loss propagation, various propagation models have been developed for predicting the propagation of radio signal in the atmosphere. These models considered series of factors limiting the propagation of radiowaves and are valuable in determining primary and 
secondary coverage areas for broadcasting stations [18]. Brief review of only three of these propagation models are presenetd in the following sub-sections.

\subsection{Okumura Model}

This model is the most frequently employed macroscopic propagation model. It was developed in mid-1960s using the data obtained from large-scale studies carried out in and around the city of Tokyo. It was designed for urban radiowave propagation environments with frequency range of $200 \mathrm{MHz}$ to $1920 \mathrm{MHz}$. The model is applicable to urban, suburban and medium urban areas.

The mathematical expression for this path loss $(P L)$ model in $\mathrm{dB}$ is expressed in $[19,20]$ as;

$P L=L_{F}+A_{m u}(f . d)-G\left(h_{t e}\right)-G\left(h_{r e}\right)-G_{\text {Area }}$

$L_{F}=20 \log _{10}\left(4 \times \pi \times d \times \frac{f}{c}\right)$

where $c$ is the speed of light in $m s^{-1}, d$ is the distance between the transmitter and receiver in $m, f$ is the frequency in $M H z, A_{m u}(f, d)$ is the function of frequency and distance, $h_{t e}$ is the height of transmitter in $m$, and $h_{r e}$ is the height of receiver in $m$. The correction factors for $G\left(h_{t e}\right), G\left(h_{r e}\right)$ and $G_{(\text {Area })}$ are defined respectively as;

$G\left(h_{t e}\right)$ is $20 \log _{10}\left(\frac{h_{t e}}{200}\right) ;$ for $h_{t e}$ between $30 \mathrm{~m}$ and $100 \mathrm{~m}$

$G\left(h_{r e}\right)$ is $20 \log _{10}\left(\frac{h_{r e}}{3}\right) ;$ for $h_{r e}$ between $3 \mathrm{~m}$ and $10 \mathrm{~m}$

$G_{(\text {Area })}$ is $\left\{\begin{array}{c}33 ; \text { for urban } \\ 27 ; \text { for suburan } \\ 13 ; \text { for medium urban }\end{array}\right.$

\subsection{Hata Model}

Hata model is an emprical model for path loss estimation or prediction. It is one of the popular propagation models usually used for radio propagation planning. It is based on an emprical relationship obtained from Okumura's report on signal strength variability measurements [21]. The model is relevant to frequencies range of $150 \mathrm{MHz}$ to $1500 \mathrm{MHz}$ with distance separation of $1 \mathrm{~km}$ to $20 \mathrm{~km}$ between the transmitter and receiver. The path loss $(P L)$ model in urban areas is expressed mathematically in $[20,21]$ as;

$P L=69.55+26.16 \log _{10} f-13.82 \log _{10} h_{t e}-a\left(h_{r e}\right)+\left(44.9-6.55 \log _{10} h_{t e}\right) \log _{10} d$

where $d, f, h_{t e}$ and $h_{r e}$ are as earlier defined. 
One of the major limitations of the predictions of this model is that, it does not consider the effect of terrain slopes and street loss. Another limitation of the model is that it does not produce good results for the hilly rural terrains due to the fact that the geographical details for hilly terrains are not provided. Thus, the model was improved to obtain COST 231-Hata model.

\subsection{COST 231-Hata Model}

COST 231-Hata model was devised as an extension to the Hata-Okumura model. The model was developed to be used in the frequency band range from $500 \mathrm{MHz}$ to $2000 \mathrm{MHz}$. However, the model contains correction factors for urban, suburban and rural or flat environments [22]. Athough this model operating frequency is outside very high frequency (VHF) band IV, it is commonly used for path loss prediction at VHF band IV or frequency modulation band because of its simplicity and the availability of correction factors [23]. This is why it was used in the comperative performance analysis conducted in section 4 of this paper. The model path loss $(P L)$ equation for COST 231-Hata model is expressed in [23, 24] as;

$P L=46.3+33.9 \log _{10} f-13.83 \log _{10} h_{b}-a h_{m}+44.9-6.55 \log _{10} h_{b} \log _{10} d+c_{m}$

where $d$ and $f$ are as earlier defined while $h_{b}$ and $h_{r}$ are the height of transmitter and receiver antennas respectively above the ground level in $m$. The correction factors $c_{m}$ and $a h_{m}$ are defined respectively as;

$c_{m}=\left\{\begin{array}{l}0 \mathrm{~dB} ; \text { for suburban or open evironment } \\ 3 \mathrm{~dB} ; \text { for urban environment }\end{array}\right.$

$a h_{m}=\left\{\begin{array}{l}\left(1.1 \log _{10} f-0.7\right) h_{r}-\left(1.56 \log _{10} f-0.8\right) ; \text { for suburban evironment } \\ 3.20\left(\log _{10} 11.7 h_{r}\right)^{2} ; \text { for urban environment }\end{array}\right.$

Observation by [24] based on Eq. 4 and Eq. 5 revealed that the $P L$ exponent of the predictions made by COST 231-Hata model is given mathematically as;

$n_{\text {COST }}=\frac{44.9-6.55 \log _{10} h_{b}}{10}$

The brief review of these three propagation models reveals that availability of correction factors are essential for direct application of model(s) developed in a particular terrain to be used in another terrain(s). The review also buttresses the findings in $[8,9]$ that there is need for different empirical models for different types of radio signals at different locations under different conditions or terrains. Furthermore, the brief review buttresses the finding reported in [25] that a radio propagation model, which gives an acceptable prediction in one location might not be suitable in another location. This implies that there is no particular propagation model that can 
directly applicable in all scenarios without the use of suitable correction factor(s). This necessitates the development of a new propagation path loss model developed in this study for Ikire metropolis. The metropolis was chosen based on the peculiarity of its topographical nature as well as the fact that no such study has been conducted in the metropolis in surveyed literature. Thus, detailed information on step-by-step activities in carrying out the study reported in this paper is presented in the next section of this paper under the research methodology.

\section{RESEARCH METHODOLOGY}

This study was conducted in Ikire metropolis of Osun State, Nigeria. Ikire lies on 7.3700 North and $4.1872^{0}$ East. The population is 143,599 according to 2006 population census. The people in the town are predominantly farmers that need quality reception of OSBC signal for their socio-economic benefits. In carrying out the study, the whole metropolis was divided into six routes: Ikire-Iwo route; Ikire-Osogbo route; Ife-Ibadan route; Post office route; Central Mosque route; and Ikire-Apomu route as shown in Fig. 1. The block diagram presented in Fig. 2 shows the activities involved in executing the study. The detailed activities in each stage in Fig. 2 are presented in the following subsections.

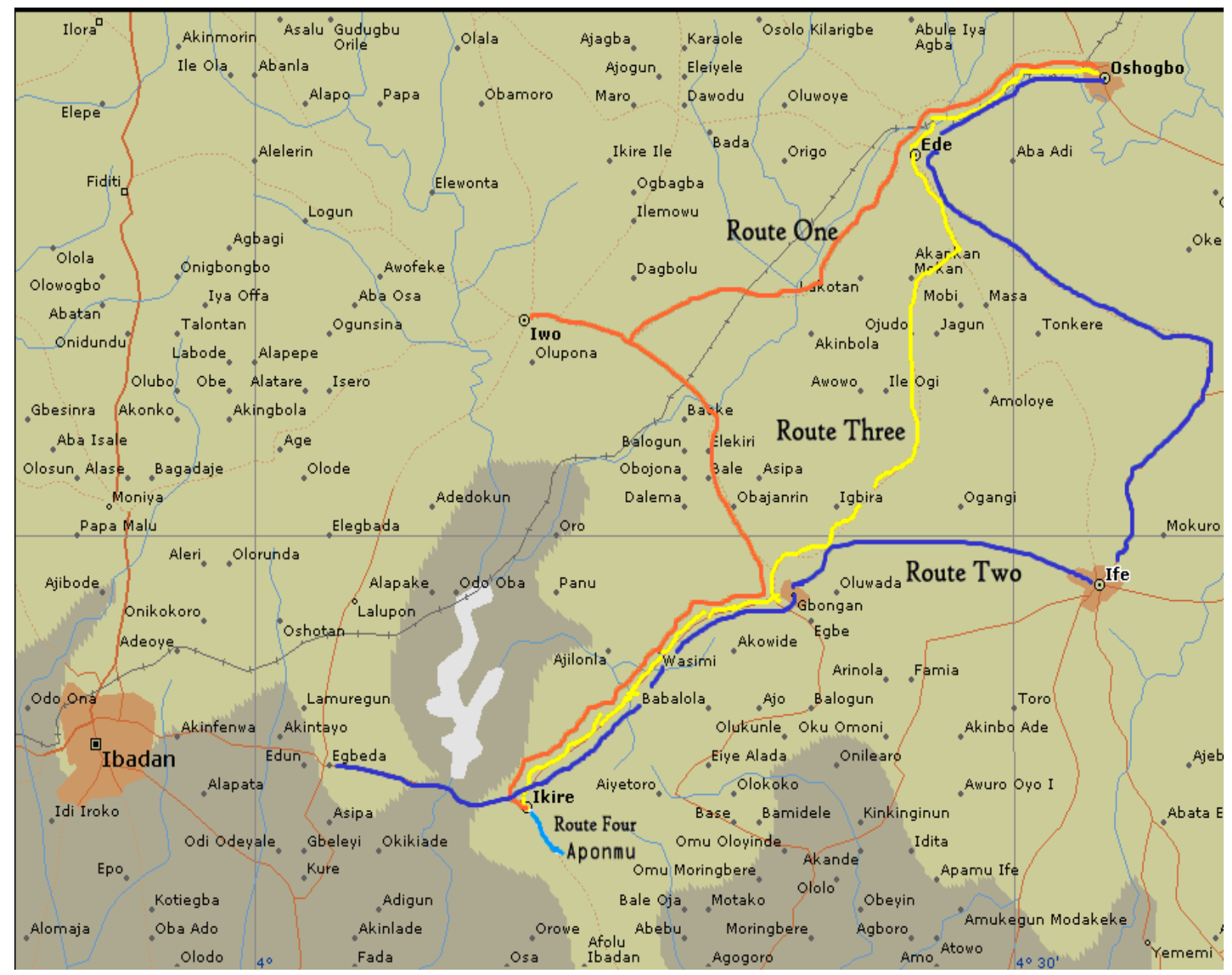

Figure 1. Map showing the field strength measurement routes 


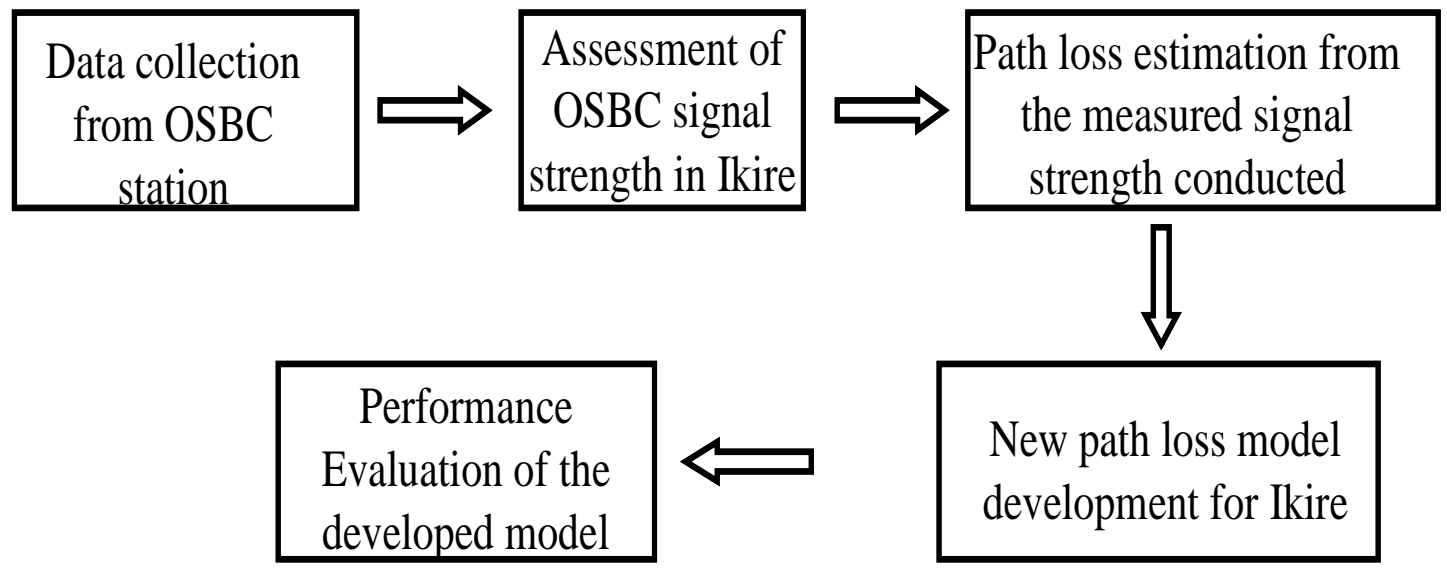

Figure 2. Block diagram of the research procedure

\subsection{Data Collection}

In this stage, characteristic data of OSBC transmitter and other related information were collected. The collected data about the Corporation are presented in Table 1.

Table 1. Characteristics data of OSBC

\begin{tabular}{ll}
\hline Parameter & Data collected \\
\hline Station location & Ile Awiye Oke-Baale, Osogbo \\
Operating frequency, $f$ & Longitude $5.2^{0}$ Latitude $7.3^{0}$ \\
Transmiited power & $104.5 \mathrm{MHz}$ \\
Power gain & $30.0 \mathrm{~kW}$ \\
Polarization & $4.83 \mathrm{~dB}$ \\
Station height above sea leave & Horizontal \\
Station antenna height & $213 \mathrm{~m}$ \\
Antenna radiation pattern & $232 \mathrm{~m}$ \\
\hline
\end{tabular}

\subsection{Experimental set-up and field measurement}

Activities in this stage involve site survey and physical planning to determine the six routes where the station signal field strength were measured. Global positioning system (GPS) and field strength meter, shown in Fig. 3(a) and Fig. 3(b) respectively were employed in measuring the required field data. While the GPS was used to determine the elevation, longitude and latitude as well as the line of sight (LOS) distance in kilometer of the transmitting antenna for each route, field strength meter was used to measure the station's radio signal at approximately $0.8 \mathrm{~km}$ apart in each route. The field strength meter used was coupled with a $75 \Omega$ dipole antenna at each observation point. The field strength measurements were conducted during the early rain season when foliage are fresh. The measurements were conducted between the hours of 7:00am and 12:00 noon when there was water vapour in the atmosphere. 


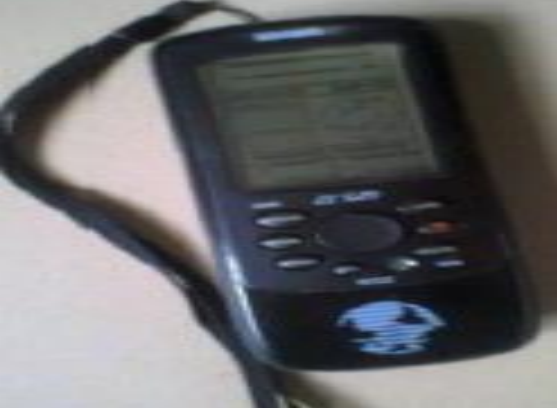

(a)

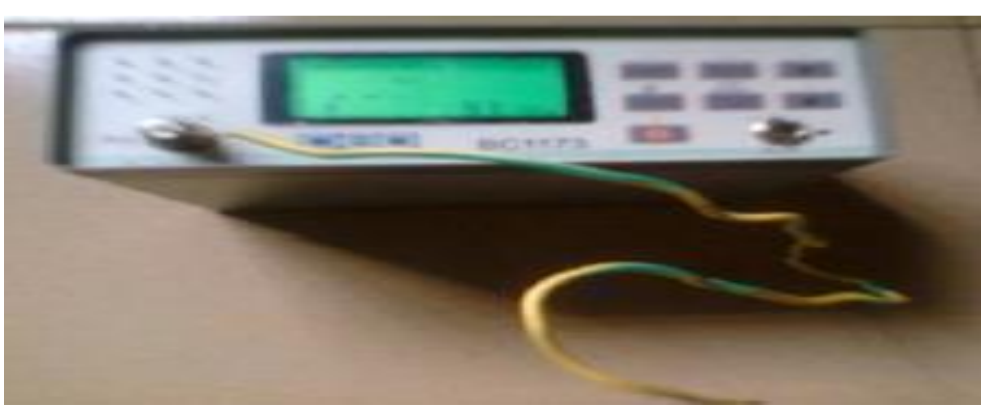

(b)

Figure 3. The used (a) GPS and (b) Field strength meter

\subsection{Path loss estimation}

The OSBC signal strength's data obtained in the last stage were compared to the estimated or theoretical values to ascertain the profile of the obtained data with the theoretical values. This was to verify the authenticity of the measured data during the radio station signal assessment as well as to determine the degree of the radio station signal degradation along each route. The mathematical expression used to estimate the theoretical values of the electric field strength, $E$, measured in $(\mathrm{V} / \mathrm{m})$, adopted from [8] is given as;

$E=\frac{\sqrt{30 P_{t} G_{t}}}{d}$

where $d$ is the LOS distance between transmitter and receiver in $\mathrm{m}, P_{t}$ is the transmitter power in watt and $G_{t}$ is the gain of the transmitter.

The path loss, $P L$, along each route was estimated using Eq. 8 and Eq. 9, which are expressed mathematically as;

$P L=\frac{P_{t} G_{t} G_{r}}{P_{r}}$

$P_{r}=\frac{G_{r} \lambda^{2} E^{2}}{4 \pi Z_{0}}$

where $\lambda$ is the wavelength, $\mathrm{Z}_{0}$ is the characteristic impedance of free space, $P_{r}$ is the received power and $G_{r}$ is the receiver gain.

The path loss along each route was computed in Microsoft Excel environment. Three out of the six plots of the path loss for the six routes were presented graphically in Fig. 4(a) - (c) due to limited space. 
Jide J. Popoola (2021). Aksaray University Journal of Science and Engineering, 5(1), 20-35.
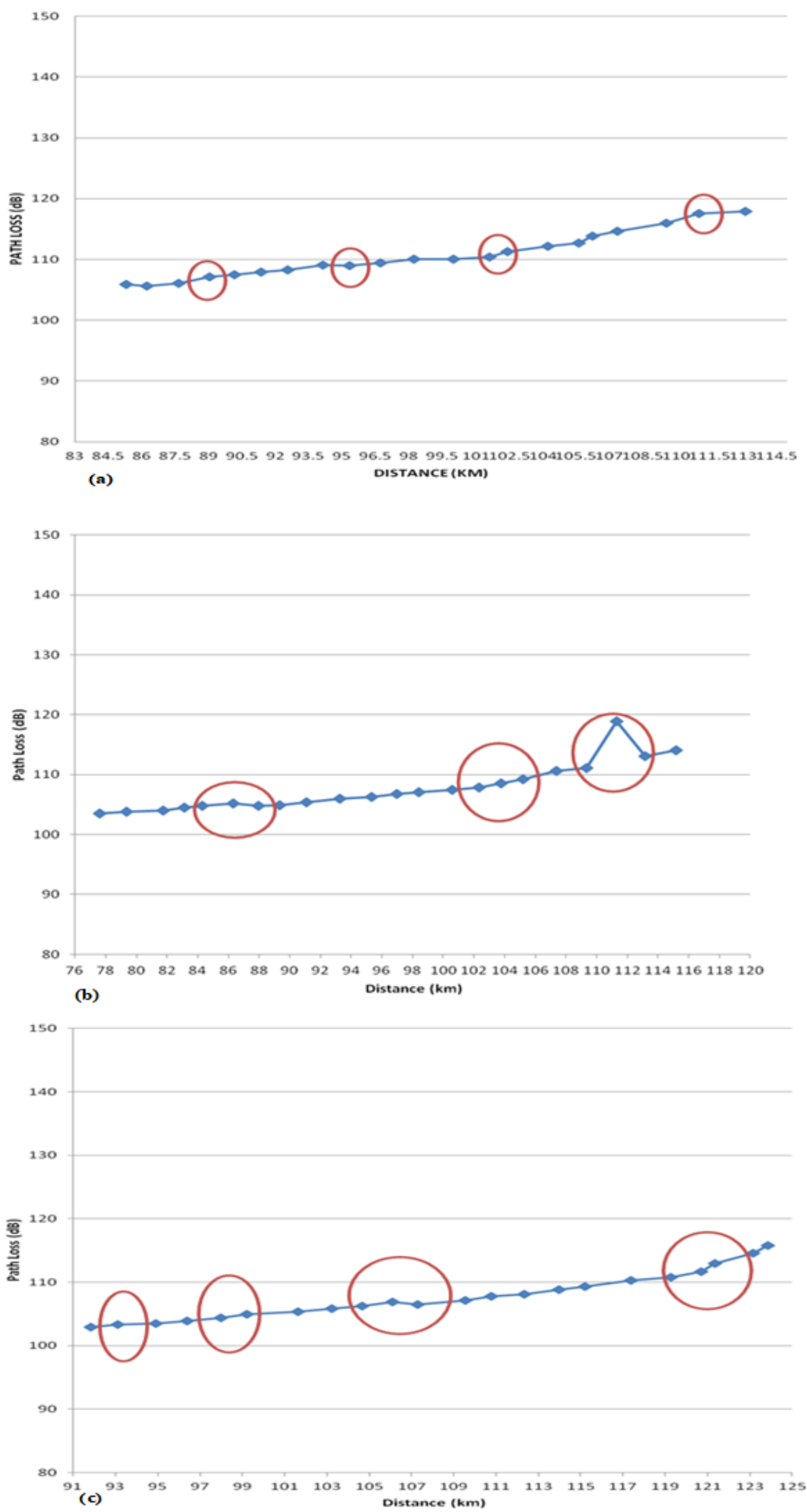

Figure 4. Estimated $P L$ for (a) Ikire-Iwo route, (b) Ife-Ibadan and (c) Ikire-Osogbo route 
Ideally, path loss usually increases as the distance between the transmitter is increasing. However, there are deviation that were marked out in red colour in Fig. 4(a)-(c). These deviations indicate need for development of an acceptable propagation model for the metropolis. Thus, the activities involved in the development of a new acceptable model for the metropolis are presented in the next subsection.

\subsection{Development of the New Path Loss Model}

The path loss propagation model for this study was developed using a least square regression test method. The method was adopted because the behaviours of the measured station's signal strength as shown in Fig. 4(a)-(c) reveal linear relationship between the estimated path loss from the study area and corresponding LOS distance in each route. The snipped picture of only one out of the six snipped pictures of the least square regression tests for the six routes onsidered is shown in Fig. 5 due to limited space.

\begin{tabular}{|c|c|c|c|c|c|c|c|c|}
\hline SUMMARY OUTPUT & & & & & & & & \\
\hline Regrecsion & Statistics & & & & & & & \\
\hline & & & & & & & & \\
\hline Multiple F & 0.976076 & & & & & & & \\
\hline R Square & 0.9527724 & & & & & & & \\
\hline Adjusted Error & 0.950236 & & & & & & & \\
\hline Standard Error & 0.824059 & & & & & & & \\
\hline Observation & 21 & & & & & & & \\
\hline & & & & & & & & \\
\hline & & & & & & & & \\
\hline ANOVA & & & & & & & & \\
\hline & df & SS & MS & $\mathrm{F}$ & Significance & & & \\
\hline & 1 & 260.0167 & 260.0167 & 282.899 & 4.74E-14 & & & \\
\hline & 19 & 12.9024 & 0.679074 & & & & & \\
\hline & 20 & 272.9191 & & & & & & \\
\hline & Coefficient & Standard Error & t Stat & P-value & Lower $95 \%$ & Upper $95 \%$ & Lower 95\% & Upper $95 \%$ \\
\hline Intercept & 69.08496 & 2.129304 & 32.44486 & $4.20 \mathrm{E}-18$ & 64.62827 & 73.54164 & 64.62827 & 73.54164 \\
\hline$X$ variable & 0.421632 & 0.021547 & 19.56781 & 4.70E-14 & 0.376534 & 0.466731 & 0.376534 & 0.466731 \\
\hline
\end{tabular}

Figure 5. Snipped picture of least square regression for developed Ikire-Iwo route $P L$ model.

Thus, the developed path loss model for Ikire-Iwo route based on the least square regression test shown in Fig. 5 is expressed as;

$P L(d B)=69.08+0.42 d$

The corresponding developed path loss models for Ife-Ibadan, Ikire-Osogbo, Post office, Central Mosque, Ikire-Apomu routes respectively based on the least square regression tests are expressed mathematically in Eqs. 11-16 as;

$P L(d B)=78.71+0.30 d$
$P L(d B)=70.26+0.35 d$ 


$$
\begin{aligned}
& P L(d B)=72.31+0.31 d \\
& P L(d B)=82.15+0.17 d \\
& P L(d B)=82.21+0.22 d
\end{aligned}
$$

The overall developed path loss model, presented in Eq. 16, for the metropolis was obtained from the average of the obtained path loss model for the six routes. The performance evaluation of the developed path loss model for the metropolis, presented in Eq. 16, was evaluated and the results obtained are presented and discussed in Section 4.

$P L(d B)=76.62+0.295 d$

where $d$ is distance in $m$.

\section{RESULTS AND DISCUSSION}

The focus of this section is on performance evaluation of the developed path loss model for this study. The section was divided into two subsections. In the first subsection, the performance evaluation of the developed model for each route was carried out. In the second subsection, the comparative performance evaluation of the developed path loss model and popular COST 231Hata model was carried out. Details on the two performance evaluation tests are presented in the following subsections.

\subsection{Performance Evaluation of the developed Model per Route}

In this performance evaluation test, the estimsted path loss obtaind using Eq. 8 and Eq. 9 and the computed path loss obtained from the developed path loss model for this study were evaluated for each of the six routes. The obtainted results were presented graphically in Figs. 6(a) - (f). Critical observations of Figs. 6(a) - (f) show that the path loss computed using the developed model for this study increases with increase in distance. This result is in agreement with the finding reported in $[26,27]$ where it was observed that foliage existing propagation models treat the forest as an effective lossy dielectric medium with exponential increase of path loss with respect to distance. The performance evaluation result per route also shows that the path loss computed using the developed path loss model for this study is relatively close to the corresponding estimated path loss value for each route. This shows that the developed path loss prediction model for this study is relatively closer to actual path loss the OSBC signal is expected to experience in each of the six routes considered if its reception will be of high quality. These results also show the effectiveness of the developed path loss prediction model for this study over the path loss model initially used in designing and planning of the OSBC 
station before its establishment, which its prediction loss was inaccurate leading to poor quality of OSBC's signal reception in the metropolis.
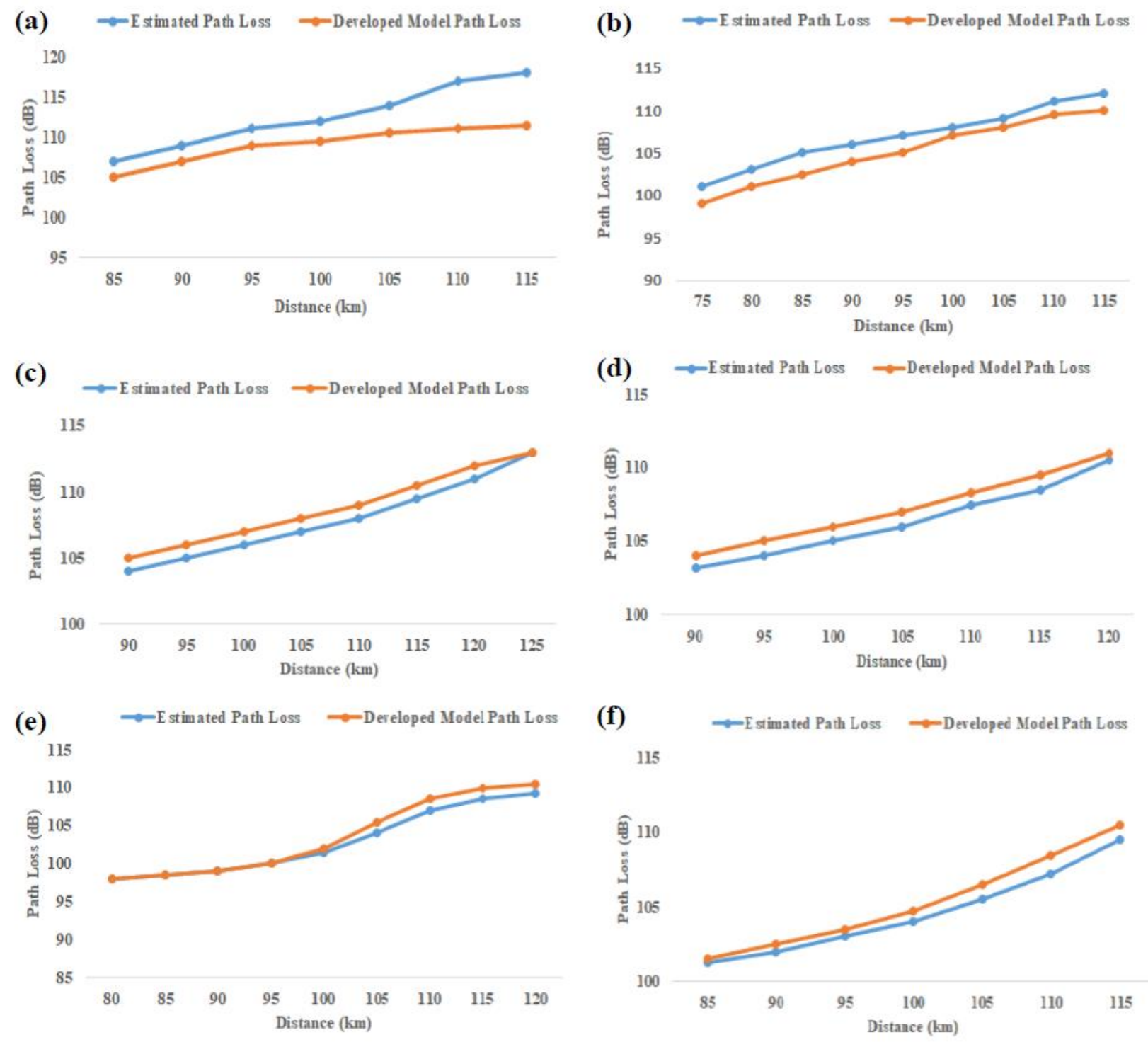

Figure 6. Developed model performance evalauation (a) Ikire-Iwo, (b) Ife-Ibadan, (c) IkireOsogbo, (d) Central Mosque, (e) Ikire-Apomu and (f) Post Office route, respectively.

\subsection{Comparative Performance Evaluation of developed Model with an existing Model}

In addition, the effectiveness of the overall performance of the developed path loss model for this study presented in Eq. 16 was further evaluated by comparing its performance efficiency with COST 231-Hata model. The existing model, COST 231-Hata, was used because of its acclained acceptability as an efficient model for predicting path loss for VHF band IV signal [23]. The result of the comparative analysis as shown graphically in Fig. 7, shows that the predicted path loss of COST 231-Hata is more than the predicted path loss by the developed path loss prediction model for this study. This indicates that the path loss prediction potential of the developed path loss model for this study performs better than that of COST 231-Hata as 
its path loss prediction value is lower than that of COST 231-Hata. This result buttresses the finding in [8], which was verified scientifically in [9] that specific path loss model ought to be developed for specific location for better quality signal reception of broadcasting signal in each location.

Furthermore, the result also shows that the estimated path loss obtained from the actual signal strength measurement conducted and the predicted path loss by the developed path loss prediction model for this study are relatively close. This indicates that the prediction potential of the developed path loss model for this study is perfectly in agreement with what the OSBC signal is experiencing during its propagation in the metropolis. The comparative performance evaluation result shown in Fig. 7 also shows that the increase in the path loss was steady as the distance increases, which confirms the suitability of the developed path loss model for the metropolis compared with COST 231-Hata used as reference model in this comparative test analysis. In addition, the overall result of this comparative performance evaluation has confirmed the discovery in [5] that development of different path loss model is necessary and essential for different areas. Likewise, these variations between the path loss predictions of the developed path loss model for this study and the COST 231-Hata used as the reference model is in agreement with the finding in [23] that, no existing model can perfectly fit in successfully into an enviornment other than where it was developed.

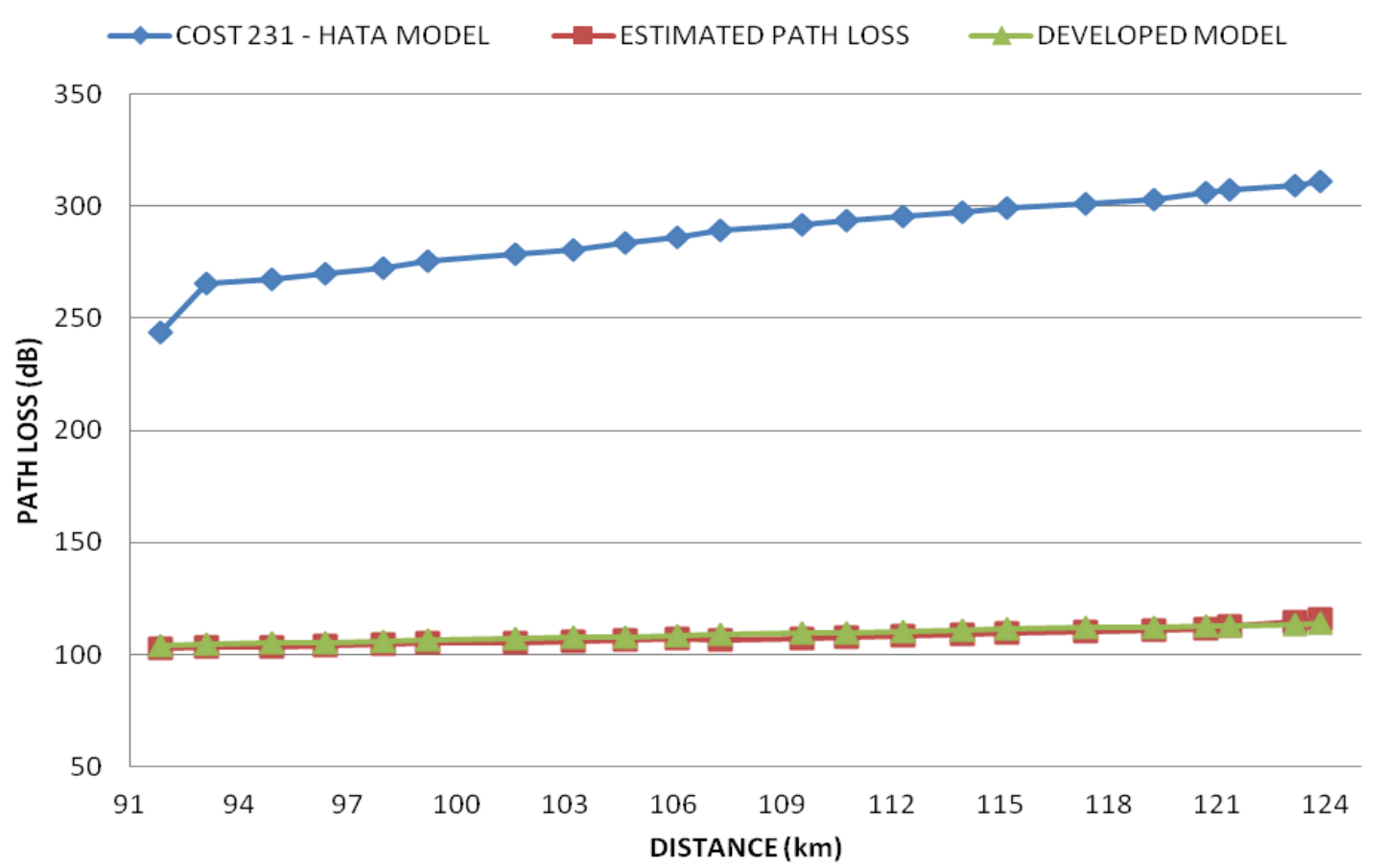

Figure 7. Developed path loss model comparative performance evaluation with COST 231Hata model 


\section{CONCLUSIONS}

This study has successfully developed a path loss prediction model applicable for Ikire metropolis of Osun State, Nigeria using OSBC broadcast signal strength measurememts conducted in six different routes within the metropolis. When the developed path loss propagation model for the metropolis was evaluated, it was found that signal strength reception is a function of distance, natural and man-made obstructions on the transmission path. It was also found that attenuation of OSBC's signal increases with increase in distance between the transmitter and receiver. Similarly, the comparative performance evaluation result obtained when the developed path loss model for this study was compared with COST 231-Hata revealed that the developed path loss model for this study performed favourably well than the reference model. The outcome of the comparative performance test therefore revealed perfectly that, different locations need different path loss models for effective and efficient signal propagation and reception of radio signal in different environments. Finally, the overall results of this study have confirmed the need for developing specific path loss prediction model for specific location or environment in order to improve signal reception quality of radio signal in specific location. Thus, one of the benefits this study will offer to researchers, broadcasting industries and the general public most especially with recent development and deployment of series of wireless communication systems, such as the fifth-generation (5G) mobile communication systems, is that it will enhance the performance optimization and coverage efficiency of these series of wireless communication systems.

\section{REFERENCES}

[1] V. Christofilakis, G. Tatsis, S.K. Chronopoulos, A. Sakkas, A.G. Skrivanos, K.P. Peppas, H.E. Nistazakis, G. Baldoumas, P. Kostarakis, Earth-to-earth microwave rain attenuation measurements: A survey on the recent literature. Symmetry. 12(9) (2020) 1-30. doi:10.3390/sym12091440.

[2] A.N. Uwaechia, N.M. Mahyuddin, A comprehensive survey on millimeter wave communications for fifth-generation wireless networks: Fesibility and Challenges. IEEE Access. 8 (2020) 6236762414. 10.1109/ACCESS.2020.2984204

[3] Y. Zhang, J. Wen, G. Yang, Z. He, J. Wang, Path loss prediction based on machine learning: Principle, method, and data expansion. Aplied Sciences. 9(9) (2019) 1-18. doi:10.3390/app9091908w.

[4] A. Ekeocha, N. Onyebuchi, A. Ifenyinwa, Comparative study of path loss models for wireless communication in urban and sub-urban environment for Port-Harcourt, Nigeria. American Journal of Engineering Research. 4(11) (2015) 109-115.

[5] D. Katiyar, V. Mittal, Implementation of cellular propagation models in diverse environments. International Journal of Engineering Trends and Technology. 15(1) (2014) 1-6. 
[6] S.I. Popoola, O.F. Oseni, Emperical path loss models for GSM networks deployment in Makurdi, Nigeria. International Refereed Journal of Engineering and Science. 3(6) (2014) 85-94.

[7] E.K. Tameh, A.R. Nix, A mix-cell propagation model for interference prediction in a UMTS network. $53^{\text {rd }}$ IEEE Vehicular Technology Conference, Rhode, Greece. (2001) 409-413.

[8] J.O. Famoriji, Y.O. Olasoji, Development of a radiowave propagation model for hilly areas. International Journal of Electronics Communication and Computer Engineering, 4(2) (2013) 536537.

[9] J.J. Popoola, A.A. Ponnle, Y.O. Olasoji, S.A. Oyetunji, Investigation of the need for specific propagation model for spsecific environmnt based on different terrain characteristics. IIUM Engineering Journal. 9(2) (2018) 90-104.

[10] O.F. Oseni, S.I. Popoola, R.O. Abolade, O.A. Adegbola, Comparative analysis of received signal strength prediction models for radio network planning of GSM $900 \mathrm{MHz}$ in Ilorin. International Journal of Innovation Technology and Exploring Engineering. 4(3) (2014) 45-50.

[11] N. Faruk, A.A. Ayeni, Y.A. Adeniran, Characterization of propagation path loss at VHF/UHF bands for Ilorin city, Nigeria. Nigerian Journal of Technology. 32(2) (2013) 253-265

[12] R. Fraile, L. Rubio, N. Carona, Application of RBF neural networks to the prediction of propagation loss over irregular terrain. $52^{\text {nd }}$ IEEE Vehicular Technology Conference, Boston, USA. (2000) 878-884.

[13] O.O. Fagbohu, Path loss determination of $91.5 \mathrm{MHz}$ FM radio channel of Ekiti State. Journal of Electrical and Electronics Engineering. 10(1) (2015) 51-57.

[14] R. Struzak, Radi-owave propagation basic. School on Digital Radio Communications for Research and Training in developing countries, the Abdus Salam International Centre for Theoretical Physics, Trieste, Italy. (2004) 1-56.

[15] Z. Stankovic, B. Milovanovic, M. Veljkovic, A Dordevic, The hybrid-neural emperical model for the electromagnetic field level prediction in urban environments. $7^{\text {th }}$ Seminar on Neural Network Applications in Electrical Engineering, Belgrade, Serba (2004) 189-192. Online [Available]: http://wireless.icp.it/school_2004/lectures/struzak/R_Prog_Basic.pdf.

[16] R. Struzak, Radi-owave propagation basic. School on Digital Radio Communications for Research and Training in developing countries, the Abdus Salam International Centre for Theoretical Physics, Trieste, Italy. (2006) 1-127. Online [Available]: http://wireless.icp.it/school_2006/lectures/Struzak/RadioProgBasic-ebook.pdf.

[17] O.O. Oyesola, Seasonal variation of mobile radio propagation characteristics in Kaduna metropolis and environs: A case study of MTN and Airtel (master Thesis in Electrical Engineering, Amadu Bello University, Zaria, Nigeria). Online [Available]: http://kubanni.abu.edu.ng:8080/jspui/handle/123456789/620.

[18] E. Ostlin, H.M. Zepernick, H. Suzuki, Evaluation of the new semi-terrain based propagation model recommendation ITU-R P.1546. 58 ${ }^{\text {th }}$ IEEE Vehicular Technology Conference, Orlando, USA. (2003) 114-118.

[19] Y. Singh, Comparison of Okumura, Hata and COST-231 models on the basis of path loss and signal strength. International Journal of Computer Applications. 59(11) (2012) 37-41. 
[20] T.P. Sarkar, Z. Ji, K. Kim, A. Medouri, M. Salazar-Palma, A survey of various propagation models for mobile communication. IEEE Antennas and Propagation Magazine. 45(3) (2003) 51-82.

[21] O.K. Ogbeide, F.O. Edeko, Modification of Hata emperical propagation model for application in $V H F$ band in Edo State, Nigeria. International Journal of Engineering Science Invention. 2(8) (2013) 35-39.

[22] G.C. Nwalozie, S.U. Ufoaroh, C.O. Ezeagwu, A.C. Ejiofor, Path loss prediction for GSM mobile networks for urban region of Aba, South-East Nigeria. International Journal of Computer Science and Mobile Computing. 3(2) (2014) 267-281.

[23] V.S. Abhayawardhana, I.J. Wassell, D. Crosby, M.P Sellars, M.G. Brown, Comparison of emperical propagation path loss models for fixed wireless access systems. $61^{\text {st }}$ IEEE Vehicular Technology Conference, Stockholm, Sweden. (2005) doi: 10.1109/VETECS.2005.1543252.

[24] R.S. Hassan, T.A. Rahman, A.Y. Abdulrahaman, LTE coverage network planning and comparison with different propagation models. TELKOMNIKA. 12(1) (2014) 153-162.

[25] C. Temaneh-Nyah, J. Nepembe, Determination of a suitable correction factor to a radio propagation model for cellular wireless network analysis. $5^{\text {th }}$ IEEE International Conference on Intelligent Systems, Modelling and Simulation, Langkawi, Malaysia. (2014) 175-182.

[26] F. Wang, I. Koh, K. Sarabandi, Long distance path-loss estimation for wave propagation through a forested evironment. IEEE International Symposium on Antennas and Propagation Society, Monterey, CA, USA. (2004) 922-925.

[27] I. Picallo, H. Klaina, P. Lopez-Iturri, E. Aguirre, M. Celaya-Echarri, L. Azpilicueta, A. Eguizabal, F. Falcone, A. Alejos, A radio channel model for D2D communications blocked by single trees in forest environments. Sensor. 19(21) (2019) 1-20. doi: 10.3390/219214606 